\title{
Computational uncertainty quantification for random non-autonomous second order linear differential equations via adapted gPC: a comparative case study with random Fröbenius method and Monte Carlo simulation
}

https://doi.org/10.1515/math-2018-0134

Received April 26, 2018; accepted December 10, 2018

\begin{abstract}
This paper presents a methodology to quantify computationally the uncertainty in a class of differential equations often met in Mathematical Physics, namely random non-autonomous second-order linear differential equations, via adaptive generalized Polynomial Chaos (gPC) and the stochastic Galerkin projection technique. Unlike the random Fröbenius method, which can only deal with particular random linear differential equations and needs the random inputs (coefficients and forcing term) to be analytic, adaptive $\mathrm{gPC}$ allows approximating the expectation and covariance of the solution stochastic process to general random second-order linear differential equations. The random inputs are allowed to functionally depend on random variables that may be independent or dependent, both absolutely continuous or discrete with infinitely many point masses. These hypotheses include a wide variety of particular differential equations, which might not be solvable via the random Fröbenius method, in which the random input coefficients may be expressed via a Karhunen-Loève expansion.
\end{abstract}

Keywords: non-autonomous and random dynamical systems, computational uncertainty quantification, adaptive generalized Polynomial Chaos, stochastic Galerkin projection technique, random Fröbenius method

MSC: 34F05, 60H35, 93E03

\section{Introduction and Preliminaries}

Many laws of Physics are formulated via differential equations. In practice, input parameters (coefficients, forcing/source term and initial/boundary conditions) of these equations are set from experimental data, thus containing the uncertainty involved in measurement errors. Furthermore, input parameters are often not exactly known because of insufficient information, limited understanding of some underlying phenomena, inherent uncertainty, etc. All these facts motivate that input parameters of classical differential equations

\footnotetext{
*Corresponding Author: Juan Carlos Cortés: Instituto Universitario de Matemática Multidisciplinar, Universitat Politècnica de València, Camino de Vera s/n, 46022, Valencia, Spain, E-mail: jccortes@imm.upv.es

Julia Calatayud: Instituto Universitario de Matemática Multidisciplinar, Universitat Politècnica de València, Camino de Vera s/n, 46022, Valencia, Spain, E-mail: jucagre@alumni.uv.es

Marc Jornet: Instituto Universitario de Matemática Multidisciplinar, Universitat Politècnica de València, Camino de Vera s/n, 46022, Valencia, Spain, E-mail: marjorsa@doctor.upv.es
} 
are treated as random variables or stochastic processes rather than deterministic constants or functions, respectively. This approach leads to random differential equations (RDEs) [1, 2]. The random behavior of the solution stochastic process can be understood if one obtains its main statistical features, say expectation, variance, covariance, etc.

A powerful tool to deal with RDEs is generalized Polynomial Chaos (gPC) [3, 4]. Let $(\Omega, \mathcal{F}, \mathbb{P})$ be a complete probability space. We will work in the Hilbert space $\left(\mathrm{L}^{2}(\Omega),\langle\cdot, \cdot\rangle\right)$ that consists of second-order random variables, i.e., random variables with finite variance, where the inner product is defined by $\left\langle\zeta_{1}, \zeta_{2}\right\rangle=\mathbb{E}\left[\zeta_{1} \zeta_{2}\right]$, being $\mathbb{E}[\cdot]$ the expectation operator. In its classical formulation, gPC consists in writing a random vector $\zeta: \Omega \rightarrow \mathbb{R}^{n}$ as a limit of multivariate polynomials evaluated at a random vector $Z: \Omega \rightarrow \mathbb{R}^{n}: \zeta \approx \sum_{i=0}^{P} \hat{\zeta}_{i} \phi_{i}(Z)$. Here $\left\{\phi_{i}(Z)\right\}_{i=0}^{\infty}$ is a sequence of orthogonal polynomials in $Z: \mathbb{E}\left[\phi_{i}(Z) \phi_{j}(Z)\right]=\int_{\mathbb{R}^{n}} \phi_{i}(z) \phi_{j}(z) \mathrm{d} \mathbb{P}_{Z}(z)=\gamma_{i} \delta_{i j}$, where $\mathbb{P}_{Z}=\mathbb{P} \circ Z^{-1}$ is the law of $Z$ and $\delta_{i j}$ is the Kronecker delta symbol. A stochastic Galerkin method can be applied to approximate the solution to RDEs [3, Ch. 6]. For some applications of this theory, see for example $[5,6]$.

Given the random vector $Z$, the sequence $\left\{\phi_{i}(Z)\right\}_{i=0}^{\infty}$ of orthogonal polynomials is taken from the AskeyWiener scheme of hypergeometric orthogonal polynomials, by taking into account the density function $f_{Z}$ of $Z$ (if $Z$ is absolutely continuous) or the discrete masses of $Z$ (if $Z$ is discrete), [3, 4].

In the recent articles [7-9], an adaptive gPC method has been developed to approximate the solutions of RDEs. Instead of taking the orthogonal polynomials from the Askey-Wiener scheme, the authors construct them directly from the random inputs that are involved in the corresponding RDE's formulation.

More explicitly, in [7], it is considered the $\operatorname{RDE} F(t, y, \dot{y})=0, y\left(t_{0}\right)=y_{0}$, where $F: \mathbb{R}^{2 q+1} \rightarrow \mathbb{R}^{q}$ and $y(t)=$ $\left(y^{1}(t), \ldots, y^{q}(t)\right)^{\top}$, where $\top$ denotes the transpose operator. The set $\left\{\zeta_{1}, \ldots, \zeta_{s}\right\}$ represents independent and absolutely continuous random input parameters in the RDE.

For each $1 \leq i \leq s$, it is considered the canonical basis of polynomials in $\zeta_{i}$ of degree at most $p$ : $\mathcal{C}_{i}^{p}=$ $\left\{1, \zeta_{i},\left(\zeta_{i}\right)^{2}, \ldots,\left(\zeta_{i}\right)^{p}\right\}$. One defines the following inner product, with weight function given by the density of $\zeta_{i}:\left\langle g\left(\zeta_{i}\right), h\left(\zeta_{i}\right)\right\rangle_{\zeta_{i}}=\int_{\mathbb{R}} g\left(\zeta_{i}\right) h\left(\zeta_{i}\right) f_{\zeta_{i}}\left(\zeta_{i}\right) \mathrm{d} \zeta_{i}$. Using a Gram-Schmidt orthonormalization procedure, one obtains a sequence of orthonormal polynomials in $\zeta_{i}$ with respect to $\langle,\rangle_{\zeta_{i}}: \Xi_{i}^{p}=\left\{\phi_{0}^{i}\left(\zeta_{i}\right), \ldots, \phi_{p}^{i}\left(\zeta_{i}\right)\right\}$. The authors build a sequence of orthonormal multivariate polynomials in $\zeta=\left(\zeta_{1}, \ldots, \zeta_{s}\right)^{\top}$ of degree at most $p$ with respect to the inner product $\langle g(\zeta), h(\zeta)\rangle_{\zeta}=\int_{\mathbb{R}^{s}} g(\zeta) h(\zeta) f_{\zeta}(\zeta) \mathrm{d} \zeta$. To do so, they build the simple tensor product $\phi_{j}(\zeta)=\phi_{p_{1}}^{1}\left(\zeta_{1}\right) \cdots \phi_{p_{s}}^{s}\left(\zeta_{s}\right), 1 \leq j \leq P$, where $j$ is associated in a bijective manner to the multi-index $\left(p_{1}, \ldots, p_{s}\right)$ in such a way that 1 corresponds to $(0, \ldots, 0)$ (for example, a graded lexicographic ordering [3, p. 66]) and $P=(p+s) ! /(p ! s !)$. By the independence between $\zeta_{1}, \ldots, \zeta_{s}$, the built sequence $\Xi=\left\{\phi_{j}(\zeta)\right\}_{j=1}^{P}$ is orthonormal with respect to $\langle,\rangle_{\zeta}$.

Once the basis is constructed, one looks for an approximate solution $y(t) \approx \sum_{j=1}^{P} y_{j}(t) \phi_{j}(\zeta)$. Then, $F\left(t, \sum_{j=1}^{P} y_{j}(t) \phi_{j}(\zeta), \sum_{j=1}^{P} \dot{y}_{j}(t) \phi_{j}(\zeta)\right)=0$. To obtain the deterministic coefficients $y_{j}(t)$, one computes the inner products $\left\langle F\left(t, \sum_{j=1}^{P} y_{j}(t) \phi_{j}(\zeta), \sum_{j=1}^{P} \dot{y}_{j}(t) \phi_{j}(\zeta)\right), \phi_{k}(\zeta)\right\rangle_{\zeta}=0, k=1, \ldots, P$. In this manner, one arrives at a deterministic system of $P$ differential equations, which may be solved by standard numerical techniques. Once $y_{1}(t), \ldots, y_{P}(t)$ have been computed, the expectation of the actual solution $y(t)$ is approximated by $y_{1}(t)$ and the covariance matrix is approximated by $\sum_{i=1}^{P} y_{i}(t) y_{i}(t)^{\top}$.

In [8], the authors use the Random Variable Transformation technique [10, Th. 1] in case that some random input parameters appearing in the RDE come from mappings of absolutely continuous random variables, whose probability density function is known.

In [9], the authors focus on the case that the random inputs $\zeta_{1}, \ldots, \zeta_{s}$ are not independent. They consider the canonical bases $\mathcal{C}_{i}^{p}=\left\{1, \zeta_{i},\left(\zeta_{i}\right)^{2}, \ldots,\left(\zeta_{i}\right)^{p}\right\}$, for $1 \leq i \leq s$, and construct a sequence of multivariate polynomials in $\zeta$, via a simple tensor product: $\phi_{j}(\zeta)=\zeta_{1}^{p_{1}} \cdots \zeta_{s}^{p_{s}}$, where $1 \leq j \leq P$ corresponds to the multiindex $\left(p_{1}, \ldots, p_{s}\right)$ and $P=(p+s) ! /(p ! s !)$. Notice that this new sequence $\left\{\phi_{j}(\zeta)\right\}_{j=1}^{P}$ is not orthonormal with respect to $\langle,\rangle_{\zeta}$. However, one proceeds with the RDE as in [7] and, in practice, one obtains good approximations of the expectation and covariance of $y(t)$.

Based on ample numerical evidence, the gPC-based methods described in [3, 4, 7-9] converge in the mean square sense at spectral rate. Some theoretical results that justify this assertion are presented in [3, pp. 33-35, p. 73], [11, Th. 2.2], [12-15]. 
In this paper we deal with an important class of differential equations with uncertainty often met in Mathematical Physics, namely general random non-autonomous second-order linear differential equations:

$$
\left\{\begin{array}{l}
\ddot{X}(t)+A(t) \dot{X}(t)+B(t) X(t)=C(t), t \in \mathbb{R} \\
X\left(t_{0}\right)=Y_{0} \\
\dot{X}\left(t_{0}\right)=Y_{1}
\end{array}\right.
$$

Our goal is to obtain approximations of the solution stochastic process $X(t)$ as well as of its main statistical features, by taking advantage of the adaptive gPC techniques [7, 9]. Here, $A(t), B(t)$ and $C(t)$ are stochastic processes and $Y_{0}$ and $Y_{1}$ are random variables in an underlying complete probability space $(\Omega, \mathcal{F}, \mathbb{P})$. The term $X(t)$ is the solution stochastic process to the random IVP (1) in some probabilistic sense. We will detail conditions for existence and uniqueness of solution in the following section.

Particular cases of (1) (with no random forcing term, $C(t)$ ) have been treated in the extant literature by using the random Fröbenius method. Specifically, Airy, Hermite, Legendre, Laguerre and Bessel differential equations have been randomized and rigorously studied in [16-21], respectively. The study includes the computation of the expectation and the variance of the solution stochastic process.

In our recent contributions [22, 23], we have studied the general problem (1) when $A(t), B(t)$ and $C(t)$ are analytic stochastic processes in the mean square sense. As it has been proved there, the random power series solution converges in the mean square sense when $A(t)$ and $B(t)$ are analytic processes in the $\mathrm{L}^{\infty}(\Omega)$ sense, $C(t)$ is a mean square convergent random power series, and the initial conditions $Y_{0}$ and $Y_{1}$ belong to $\mathrm{L}^{2}(\Omega)$. Under those assumptions, the expectation and variance statistics of the solution process $X(t)$ can be rigorously approximated.

In [24] the authors study RDEs by taking advantage of homotopy analysis and they provide a complete set of illustrative examples dealing with random second-order linear differential equations.

In this paper, we want to go one step further and we will perform a computational analysis based upon adaptive gPC, by showing its capability to deal with the general random IVP (1) that comprises Airy, Hermite, Legendre, Laguerre and Bessel differential equations, or any other formulation of (1) based on analytic data processes, just as particular cases. We will thus resolve the future line of research brought up in [23, Section 5].

The paper is organized as follows. Section 2 describes the application of adaptive gPC to solve the random IVP (1) and the computation of the expectation and covariance of $X(t)$. The study is split into two cases depending on the probabilistic dependence of the random inputs. In Section 3, we show the algorithms corresponding to the theory previously developed in Section 2. Section 4 is addressed to show particular examples of (1) where adaptive gPC, Fröbenius method and Monte Carlo simulation are carried out to obtain approximations for the expectation, variance and covariance of the solution stochastic process. It is evinced that adaptive gPC provides the same results as the Fröbenius method with small orders of basis $p$, and, moreover, in cases where the Fröbenius method is not applicable, adaptive gPC might be successful. Finally, in Section 5, conclusions are drawn.

\section{Method}

Consider the random IVP (1), where

$$
A(t)=a_{0}(t)+\sum_{i=1}^{d_{A}} a_{i}(t) \gamma_{i}, B(t)=b_{0}(t)+\sum_{i=1}^{d_{B}} b_{i}(t) \eta_{i}, C(t)=c_{0}(t)+\sum_{i=1}^{d_{C}} c_{i}(t) \xi_{i},
$$

being $\gamma_{1}, \ldots, \gamma_{d_{A}}, \eta_{1}, \ldots, \eta_{d_{B}}$ and $\xi_{1}, \ldots, \xi_{d_{C}}$ random variables (not necessarily independent) and $a_{0}(t), \ldots, a_{d_{A}}(t), b_{0}(t), \ldots, b_{d_{B}}(t)$ and $c_{0}(t), \ldots, c_{d_{C}}(t)$ real functions. Representation (2) for the input stochastic processes includes truncated random power series [2, p. 99] and Karhunen-Loève expansions [3, Ch. 4], [25, Ch. 5]. This is an improvement with respect to the random Fröbenius method used in [16-23], in which $A(t), B(t)$ and $C(t)$ are only expressed as random power series. 
As we are interested in constructive computational aspects of uncertainty quantification, we will assume that there exists a unique solution stochastic process $X(t)$ to IVP (1) in some probabilistic sense, for instance, sample path [1, SP problem] [2, Appendix A], or $\mathrm{L}^{q}(\Omega)$ sense [2], in such a way that $\mathbb{E}\left[X(t)^{2}\right]<\infty$ for each $t$. We detail the conditions under which there exists a unique solution $X(t)$ to (1) in the following propositions. The proofs are simple consequences of the references cited therein. Proposition 2.1, which is concerned with sample path solutions, is a direct consequence of the deterministic theory on ordinary differential equations (Carathéodory theory on the existence of absolutely continuous solutions [26, pp. 28-30]). Proposition 2.2 takes advantage of a natural generalization to $\mathrm{L}^{q}(\Omega)$ random calculus of the classical Picard theorem for deterministic ordinary differential equations [2, Th. 5.1.2].

Proposition 2.1 (Sample path solution). [26, pp. 28-30] If $A(t), B(t)$ and $C(t)$ have real integrable sample paths, then there exists a unique solution stochastic process $X(t)$ to (1) with $C^{1}$ sample paths and derivative $\dot{X}(t)$ with absolutely continuous sample paths (i.e., $X(t)$ is a classical solution that belongs to the Sobolev space $\left.W^{2,1}\right)$. Moreover, if $A(t), B(t)$ and $C(t)$ have continuous sample paths, then $X(t)$ has $C^{2}$ sample paths.

Proposition 2.2 ( $\mathrm{L}^{q}(\Omega)$ solution). [2, Ch. 5], [23] If $A(t)$ and $B(t)$ are continuous stochastic processes in the $\mathrm{L}^{\infty}(\Omega)$ sense, and the source term $C(t)$ is continuous in the $\mathrm{L}^{q}(\Omega)$ setting, then there exists a unique solution $X(t)$ to (1) in the $\mathrm{L}^{q}(\Omega)$ sense.

Our goal is to approximate the solution stochastic process $X(t)$ to the random IVP (1) by using adaptive gPC, which is described in [7, 9] and has been reviewed in Section 1. In the case that the random inputs $\gamma_{1}, \ldots, \gamma_{d_{A}}, \eta_{1}, \ldots, \eta_{d_{B}}, \xi_{1}, \ldots, \xi_{d_{C}}, Y_{0}$ and $Y_{1}$ are independent, we will use the method from [7], whereas in the case that they are not independent, [9] will be utilized. In [7, 9], the random inputs are assumed to be absolutely continuous, so that the weights in the inner products are given by density functions. Notice, however, that a discrete distribution with infinitely many point masses can be given to the random inputs. Indeed, the corresponding inner product becomes an integral with respect to a discrete law, which is a series with weights being the probabilities of the point masses. Moreover, since the support has infinite cardinality, the corresponding canonical basis of polynomials has infinite dimension, so that its length $p$ can grow up to infinity.

For ease of notation and to identify the notation with the one used in Section 1, we denote the random inputs $\gamma_{1}, \ldots, \gamma_{d_{A}}, \eta_{1}, \ldots, \eta_{d_{B}}, \xi_{1}, \ldots, \xi_{d_{C}}, Y_{0}$ and $Y_{1}$ as $\zeta_{1}, \ldots, \zeta_{s}$, where $s=d_{A}+d_{B}+d_{C}+2$. The random variables $\zeta_{1}, \ldots, \zeta_{s}$ are not necessarily independent, and they are absolutely continuous or discrete random variables with infinitely many point masses. We will denote $\zeta=\left(\zeta_{1}, \ldots, \zeta_{s}\right)^{\top}$. The space of polynomials evaluated at $\zeta_{i}$ of degree at most $p$ will be denoted by $\mathcal{P}_{p}\left[\zeta_{i}\right]$. The space of multivariate polynomials evaluated at $\zeta$ of degree at most $P$ will be written as $\mathcal{P}_{P}^{S}[\zeta]$.

In the next development, we distinguish two cases depending on whether the random inputs $\zeta_{1}, \ldots, \zeta_{s}$ are independent or not.

\subsection{The random inputs are independent}

In the notation from [7] and Section 1 , let $\mathcal{C}_{i}^{p}=\left\{1, \zeta_{i}, \ldots, \zeta_{i}^{p}\right\}$ be the canonical basis of $\mathcal{P}_{p}\left[\zeta_{i}\right]$, for $i=1, \ldots, s$. Let $\Xi_{i}^{p}=\left\{\phi_{0}^{i}\left(\zeta_{i}\right), \ldots, \phi_{p}^{i}\left(\zeta_{i}\right)\right\}$ be the orthonormalization of $\mathcal{C}_{i}^{p}$ with respect to the inner product defined by the law $\mathbb{P}_{\zeta_{i}}$, via a Gram-Schmidt procedure. Let $\Xi=\left\{\phi_{1}(\zeta), \ldots, \phi_{P}(\zeta)\right\}$ be the orthonormal basis of $\mathcal{P}_{P}^{s}[\zeta]$ with respect to the law $\mathbb{P}_{\zeta}=\mathbb{P}_{\zeta_{1}} \times \cdots \times \mathbb{P}_{\zeta_{s}}$, where $P=(p+s) ! /(p ! s !)$.

We approximate the solution stochastic process $X(t) \approx \sum_{i=1}^{P} x_{i}(t) \phi_{i}(\zeta)$ by imposing the right-hand side to be a solution to random IVP (1):

$$
\sum_{i=1}^{P} \ddot{x}_{i}(t) \phi_{i}(\zeta)+\left(a_{0}(t)+\sum_{i=1}^{d_{A}} a_{i}(t) \gamma_{i}\right)\left(\sum_{i=1}^{P} \dot{x}_{i}(t) \phi_{i}(\zeta)\right)
$$




$$
+\left(b_{0}(t)+\sum_{i=1}^{d_{B}} b_{i}(t) \eta_{i}\right)\left(\sum_{i=1}^{P} x_{i}(t) \phi_{i}(\zeta)\right)=c_{0}(t)+\sum_{i=1}^{d_{C}} c_{i}(t) \xi_{i} .
$$

We apply the stochastic Galerkin projection technique. By multiplying by $\phi_{k}(\zeta), k=1, \ldots, P$, applying expectations, using the orthonormality of $\Xi$ and the fact that $\phi_{1}=1$, we obtain:

$$
\begin{gathered}
\ddot{x}_{k}(t)+a_{0}(t) \dot{x}_{k}(t)+\sum_{i=1}^{d_{A}} \sum_{j=1}^{P} a_{i}(t) \dot{x}_{j}(t) \mathbb{E}\left[\gamma_{i} \phi_{j}(\zeta) \phi_{k}(\zeta)\right]+b_{0}(t) x_{k}(t) \\
+\sum_{i=1}^{d_{B}} \sum_{j=1}^{P} b_{i}(t) x_{j}(t) \mathbb{E}\left[\eta_{i} \phi_{j}(\zeta) \phi_{k}(\zeta)\right]=c_{0}(t) \delta_{1 k}+\sum_{i=1}^{d_{C}} c_{i}(t) \mathbb{E}\left[\xi_{i} \phi_{k}(\zeta)\right] .
\end{gathered}
$$

Let us put this equation in matrix form. Consider the $P \times P$ matrices $M$ and $N$ defined by

$$
M_{k j}(t)=\sum_{i=1}^{d_{A}} a_{i}(t) \mathbb{E}\left[\gamma_{i} \phi_{j}(\zeta) \phi_{k}(\zeta)\right], \quad N_{k j}(t)=\sum_{i=1}^{d_{B}} b_{i}(t) \mathbb{E}\left[\eta_{i} \phi_{j}(\zeta) \phi_{k}(\zeta)\right],
$$

for $k, j=1, \ldots, P$. Consider the vector $q$ of length $P$ with

$$
q_{k}=\sum_{i=1}^{d_{C}} c_{i}(t) \mathbb{E}\left[\xi_{i} \phi_{k}(\zeta)\right]
$$

for $k=1 \ldots, P$. We rewrite (4) as a deterministic system of $P$ differential equations:

$$
\ddot{x}(t)+\left(M(t)+a_{0}(t) I_{P}\right) \dot{x}(t)+\left(N(t)+b_{0}(t) I_{p}\right) x(t)=q(t)+c_{0}(t) e_{1},
$$

where $x(t)=\left(x_{1}(t), \ldots, x_{P}(t)\right)^{\top}, I_{P}$ is the $P \times P$ identity matrix and $e_{1}$ is the first vector of the canonical basis: $(1,0, \ldots, 0)^{\top}$. It remains to find the initial condition for (7). From $\sum_{i=1}^{P} x_{i}\left(t_{0}\right) \phi_{i}(\zeta)=Y_{0}$ and $\sum_{i=1}^{P} \dot{x}_{i}\left(t_{0}\right) \phi_{i}(\zeta)=Y_{1}$, we obtain that $x_{k}\left(t_{0}\right)=\mathbb{E}\left[Y_{0} \phi_{k}(\zeta)\right]$ and $\dot{x}_{k}\left(t_{0}\right)=\mathbb{E}\left[Y_{1} \phi_{k}(\zeta)\right]$, for $k=1, \ldots, P$. Thus, the initial conditions become $x\left(t_{0}\right)=y$ and $\dot{x}\left(t_{0}\right)=y^{\prime}$, where $y=\left(y_{1}, \ldots, y_{P}\right)^{\top}$ and $y^{\prime}=\left(y_{1}^{\prime}, \ldots, y_{P}^{\prime}\right)^{\top}$,

$$
y_{k}=\mathbb{E}\left[Y_{0} \phi_{k}(\zeta)\right], \quad y_{k}^{\prime}=\mathbb{E}\left[Y_{1} \phi_{k}(\zeta)\right],
$$

for $k=1, \ldots, P$.

The system of deterministic differential equations can be solved by using standard numerical techniques. Once we have computed the solution $\left(x_{1}(t), \ldots, x_{P}(t)\right)$, we have obtained the approximation $\sum_{i=1}^{P} x_{i}(t) \phi_{i}(\zeta)$ for the solution stochastic process $X(t)$. Moreover, one can approximate the expectation and covariance of $X(t)$ :

$$
\mathbb{E}[X(t)] \approx x_{1}(t), \quad \operatorname{Cov}\left[X\left(t_{1}\right), X\left(t_{2}\right)\right] \approx \sum_{i=2}^{P} x_{i}\left(t_{1}\right) x_{i}\left(t_{2}\right) .
$$

\subsection{The random inputs may not be independent}

In the notation from [9] and Section 1 , let $\mathcal{C}_{i}^{p}=\left\{1, \zeta_{i}, \ldots, \zeta_{i}^{p}\right\}$ be the canonical basis of $\mathcal{P}_{p}\left[\zeta_{i}\right]$, for $i=1, \ldots, s$. We construct the basis $\Xi=\left\{\phi_{1}, \ldots, \phi_{P}\right\}$ of $\mathcal{P}_{P}^{S}[\zeta]$ as in [9]. This basis is not orthonormal with respect to the law $\mathbb{P}_{\zeta}$.

We approximate the solution stochastic process $X(t) \approx \sum_{i=1}^{P} x_{i}(t) \phi_{i}(\zeta)$ by imposing the right-hand side to be a solution to random IVP (1). One obtains (3). By multiplying by $\phi_{k}(\zeta)$ and applying expectations, $k=$ $1, \ldots, P$, we derive that

$$
\sum_{i=1}^{P} \ddot{x}_{i}(t) \mathbb{E}\left[\phi_{i}(\zeta) \phi_{k}(\zeta)\right]+a_{0}(t) \sum_{i=1}^{P} \dot{x}_{i}(t) \mathbb{E}\left[\phi_{i}(\zeta) \phi_{k}(\zeta)\right]
$$




$$
\begin{aligned}
& +\sum_{i=1}^{d_{A}} \sum_{j=1}^{P} a_{i}(t) \dot{x}_{j}(t) \mathbb{E}\left[\gamma_{i} \phi_{j}(\zeta) \phi_{k}(\zeta)\right]+b_{0}(t) \sum_{i=1}^{P} x_{i}(t) \mathbb{E}\left[\phi_{i}(\zeta) \phi_{k}(\zeta)\right] \\
& +\sum_{i=1}^{d_{B}} \sum_{j=1}^{P} b_{i}(t) x_{j}(t) \mathbb{E}\left[\eta_{i} \phi_{j}(\zeta) \phi_{k}(\zeta)\right] \\
& =c_{0}(t) \mathbb{E}\left[\phi_{k}(\zeta)\right]+\sum_{i=1}^{d_{C}} c_{i}(t) \mathbb{E}\left[\xi_{i} \phi_{k}(\zeta)\right] .
\end{aligned}
$$

Define the $P \times P$ matrix $R$ and the vector $h$ of length $P$ as

$$
R_{i k}=\mathbb{E}\left[\phi_{i}(\zeta) \phi_{k}(\zeta)\right], \quad h_{k}=\mathbb{E}\left[\phi_{k}(\zeta)\right],
$$

for $i, k=1, \ldots, P$. Expression (10) can be written in matrix form as a deterministic system of $P$ differential equations:

$$
R \ddot{x}(t)+\left(M(t)+a_{0}(t) R\right) \dot{x}(t)+\left(N(t)+b_{0}(t) R\right) x(t)=q(t)+c_{0}(t) h .
$$

The initial conditions are given by $R x\left(t_{0}\right)=y$ and $R \dot{x}\left(t_{0}\right)=y^{\prime}$.

This system of deterministic differential equations is solvable by standard numerical techniques. Once we have computed the approximation $\sum_{i=1}^{P} x_{i}(t) \phi_{i}(\zeta)$ of the solution stochastic process $X(t)$, the expectation and covariance of $X(t)$ can be approximated as follows:

$$
\mathbb{E}[X(t)] \approx \sum_{i=1}^{P} x_{i}(t) \mathbb{E}\left[\phi_{i}(\zeta)\right], \operatorname{Cov}\left[X\left(t_{1}\right), X\left(t_{2}\right)\right] \approx \sum_{i=1}^{P} \sum_{j=1}^{P} x_{i}\left(t_{1}\right) x_{j}\left(t_{2}\right) \operatorname{Cov}\left[\phi_{i}(\zeta), \phi_{j}(\zeta)\right] .
$$

\section{Algorithm}

In this section we present the algorithm corresponding to Section 2. From the random inputs $A(t), B(t)$ and $C(t)$ having expression (2) and the initial conditions $Y_{0}$ and $Y_{1}$, we will show the steps to be followed in order to approximate the expectation and covariance of the solution stochastic process $X(t)$. As in Section 2, denote the random input parameters by $\zeta_{1}, \ldots, \zeta_{s}$.

Case $\zeta_{1}, \ldots, \zeta_{s}$ are independent:

Step 1. Define the canonical basis $\mathcal{C}_{i}^{p}=\left\{1, \zeta_{i}, \ldots, \zeta_{i}^{p}\right\}, i=1, \ldots, s$.

Step 2. Via a Gram-Schmidt procedure, orthonormalize $\mathcal{C}_{i}^{p}$ to a new basis $\Xi_{i}^{p}=\left\{\phi_{0}^{i}(\zeta), \ldots, \phi_{p}^{i}(\zeta)\right\}$ with respect to the probability law $\mathbb{P}_{\zeta_{i}}$ of $\zeta_{i}$. In the software Mathematica ${ }^{\circledR}$, this can be readily done with the built-in function Orthogonalize. For example, if $p=3$ and the probability distribution is dist, then the command could be:

Expand [Orthogonalize $[\{1, \mathrm{Z}, \mathrm{Z} \sim 2, \mathrm{Z}$ 3 $\}$,

Integrate [\#1 \#2 PDF [dist, Z], \{Z, -Infinity, Infinity $\}]$ \&]]

Step 3. By using a simple tensor product, define the orthonormal basis with respect to the joint law $\mathbb{P}_{\zeta}=$ $\mathbb{P}_{\zeta_{1}} \times \cdots \times \mathbb{P}_{\zeta_{s}}, \Xi=\left\{\phi_{1}(\zeta), \ldots, \phi_{P}(\zeta)\right\}$

Step 4. Construct the matrices $M(t)$ and $N(t)$ given by (5), the vector $q(t)$ defined by (6), and the initial conditions $y$ and $y^{\prime}$ given by (8). All the involved expectations can be calculated with the built-in function Expectation from Mathematica ${ }^{\circledR}$.

Step 5. Solve numerically the deterministic system of $P$ differential equations given by (7) with initial conditions $x\left(t_{0}\right)=y$ and $\dot{x}\left(t_{0}\right)=y^{\prime}$. This system does not pose serious numerical challenges. We thus integrate the equations over time with the standard NDSolve routine from Mathematica ${ }^{\circledR}$ : write the instruction NDSolve [eqns, function, $\{t, t 0, T\}]$

with automatic method, step size, etc. (the built-in function will automatically try to estimate the best method for a particular computation). 
Step 6. Approximate the expectation and covariance of the unknown solution stochastic process by using (9).

Case $\zeta_{1}, \ldots, \zeta_{s}$ are not independent:

Step 1. Define the canonical basis $\mathcal{C}_{i}^{p}=\left\{1, \zeta_{i}, \ldots, \zeta_{i}^{p}\right\}, i=1, \ldots, s$.

Step 2. By using a simple tensor product, define the basis $\Xi=\left\{\phi_{1}(\zeta), \ldots, \phi_{P}(\zeta)\right\}$.

Step 3. Construct the matrices $M(t)$ and $N(t)$ given by (5), the vector $q(t)$ defined by (6), the matrix $R(t)$ and the vector $h$ given by (11), and the vectors $y$ and $y^{\prime}$ expressed by (8). All the involved expectations can be calculated with the built-in function Expectation from Mathematica ${ }^{\circledast}$.

Step 4. Solve numerically the deterministic system of $P$ differential equations given by (12) with initial conditions $R x\left(t_{0}\right)=y$ and $R \dot{x}\left(t_{0}\right)=y^{\prime}$. This system does not pose serious numerical challenges. We thus integrate the equations over time with the standard NDSolve routine from Mathematica ${ }^{\circledR}$ with the option

Method -> \{"EquationSimplification" -> "Residual"\}

(to deal with the corresponding system of differential-algebraic equations): write the instruction

NDSolve [eqns, function, $\{t, t 0, T\}$,

Method $\rightarrow$ \{"EquationSimplification" -> "Residual"\}]

with automatic method, step size, etc. (the built-in function will automatically try to pick the best method for a particular computation).

Step 5. Approximate the expectation and covariance of the unknown solution stochastic process by using (13).

\section{Examples}

In this section we show particular examples of the random IVP (1) to which we apply adaptive gPC to approximate the expectation and covariance of the solution stochastic process $X(t)$.

We will compare the results with Monte Carlo simulation. This method is based on sampling. Sample from the probability distributions of $A(t), B(t), C(t), Y_{0}$ and $Y_{1}$ to obtain, say $m$ realizations, for $m$ large:

$$
\begin{gathered}
A^{(1)}(t), \ldots, A^{(m)}(t), B^{(1)}(t), \ldots, B^{(m)}(t), C^{(1)}(t), \ldots, C^{(m)}(t), \\
Y_{0}^{(1)}, \ldots, Y_{0}^{(m)}, Y_{1}^{(1)}, \ldots, Y_{1}^{(m)} .
\end{gathered}
$$

Then we solve the $m$ deterministic initial value problems

$$
\left\{\begin{array}{l}
\ddot{X}^{(i)}(t)+A^{(i)}(t) \dot{X}^{(i)}(t)+B^{(i)}(t) X^{(i)}(t)=C^{(i)}(t), t \in \mathbb{R} \\
X^{(i)}\left(t_{0}\right)=Y_{0}^{(i)} \\
\dot{X}^{(i)}\left(t_{0}\right)=Y_{1}^{(i)}
\end{array}\right.
$$

so that we obtain $m$ realizations of $X(t): X^{(1)}(t), \ldots, X^{(m)}(t)$. The Law of Large Numbers permits approximating $\mathbb{E}[X(t)]$ and $\mathbb{V}[X(t)]$ by computing the sample mean and sample variance of $X^{(1)}(t), \ldots, X^{(m)}(t)$ :

$$
\mathbb{E}[X(t)] \approx \mu_{m}(t)=\frac{1}{m} \sum_{i=1}^{m} X^{(i)}(t), \quad \mathbb{V}[X(t)] \approx \frac{1}{m-1} \sum_{i=1}^{m}\left(X^{(i)}(t)-\mu_{m}(t)\right)^{2} .
$$

The results of adaptive gPC agree with Monte Carlo simulation, although the convergence rate of Monte Carlo is much slower (its error convergence rate is inversely proportional to the square root of the number of realizations [3, p. 53]).

The result of the expectation will also be compared with the dishonest method [27, p. 149]. It consists in estimating $\mathbb{E}[X(t)]$ by substituting $A(t), B(t), C(t), Y_{0}$ and $Y_{1}$ in (1) by their corresponding expected values. Denoting $\mu_{X}(t)=\mathbb{E}[X(t)]$, the idea is that, since $\mathbb{E}[\ddot{X}(t)]=\frac{\mathrm{d}^{2}}{\mathrm{~d} t^{2}}\left(\mu_{X}(t)\right)$ and $\mathbb{E}[\dot{X}(t)]=\frac{\mathrm{d}}{\mathrm{d} t}\left(\mu_{X}(t)\right)$, because of the 
commutation between the mean square limit and the expectation operator (see [2, Ch. 4]), one solves:

$$
\left\{\begin{array}{l}
\frac{\mathrm{d}^{2}}{\mathrm{~d} t^{2}}\left(\mu_{X}(t)\right)+\mathbb{E}[A(t)] \frac{\mathrm{d}}{\mathrm{d} t}\left(\mu_{X}(t)\right)+\mathbb{E}[B(t)] \mu_{X}(t)=\mathbb{E}[C(t)], t \in \mathbb{R}, \\
\mu_{X}\left(t_{0}\right)=\mathbb{E}\left[Y_{0}\right] \\
\frac{\mathrm{d}}{\mathrm{d} t}\left(\mu_{X}\left(t_{0}\right)\right)=\mathbb{E}\left[Y_{1}\right] .
\end{array}\right.
$$

In our context, the dishonest method will work on cases where $\operatorname{Cov}[A(t), \dot{X}(t)]$ and $\operatorname{Cov}[B(t), X(t)]$ are small, but in general, there is no certainty that this may hold. Thus, this method is a naive approximation to the true expectation, with no theoretical support, although with a certain use in the literature [27].

When possible, the results obtained via adaptive gPC for the expectation and variance will be compared with the random Fröbenius method. The convergence of the random Fröbenius method will be guaranteed by previous studies, see [22, 23].

Several conclusions are drawn from these examples. Adaptive gPC allows for random inputs (2) more general than the random Fröbenius method: $A(t), B(t)$ and $C(t)$ may not be analytic, they may be represented via a truncated Karhunen-Loève expansion, etc. Moreover, with a small length $p$ of the bases, accurate results are obtained (this is due to the well-known spectral convergence of gPC-based methods). In practical applications, a disadvantage of adaptive $\mathrm{gPC}$ is that random parameter inputs cannot have a finite number of point masses (otherwise the space of polynomials evaluated at them would have finite dimension). From a computational standpoint, a large number $s$ of random input parameters may make the computations inviable, as the order $P$ of the basis increases as $P=(p+s) ! /(p ! s !)$.

Example 4.1. Airy-type differential equations appear in a variety of applications to Mathematical Physics, such as the description of the solution to the Schrödinger equation for a particle confined within a triangular potential, in the solution for the one-dimensional motion of a quantum particle affected by a constant force, or in the theory of diffraction of radio waves around the Earth's surface [28]. Airy's random differential equation is given by [16]:

$$
\left\{\begin{array}{l}
\ddot{X}(t)+\operatorname{AtX}(t)=0, t \in \mathbb{R} \\
X(0)=Y_{0} \\
\dot{X}(0)=Y_{1}
\end{array}\right.
$$

where $A, Y_{0}$ and $Y_{1}$ are random variables. It is well-known that the solution to the deterministic Airy's differential equation is highly oscillatory, hence it is expected that, in dealing with its stochastic counterpart, differences between distinct methods will be highlighted.

Existence and uniqueness of sample path solution is guaranteed by Proposition 2.1. Concerning the existence and uniqueness of mean square solution, we refer to [16, 22] or Proposition 2.2, under the assumption that $A$ is a bounded random variable. This assumption on boundedness is not a restriction in practice, as one may truncate the random variable $A$ with a support as large as desired.

In [16], the following distributions for $A, Y_{0}$ and $Y_{1}$ are set: $A \sim \operatorname{Beta}(2,3), Y_{0} \sim \operatorname{Normal}(1,1)$ and $Y_{1} \sim \operatorname{Normal}(2,1)$. They are assumed to be independent. Approximations for the expectation and variance via the random Fröbenius method and Monte Carlo simulation are obtained in [16]. We use adaptive gPC (independent case) with $p=3, p=4, \zeta_{1}=A, \zeta_{2}=Y_{0}$ and $\zeta_{3}=Y_{1}, \eta_{1}=A, A(t)=0, C(t)=0$ and $b_{1}(t)=t$. The results obtained are shown in Table 1 (expectation), Table 2 (variance) and Table 3 (covariance). The order of truncation in the random Fröbenius method is denoted by $N$. Observe that gPC expansions have converged for $t \in[0,2]$ with order $p=3$. This rapid convergence shows the potentiality of this approach.

In Figure 1, we focus on the convergence of gPC expansions. The solid line reflects the expectations, while the dashed lines represent confidence intervals constructed with the rule mean \pm deviation (the standard deviation stands for the square root of the variance). Observe that, as we move away from $t=0$, larger orders of $p$ are required to achieve good approximations of the statistics of $X(t)$. Indeed, Galerkin projections deviate from the exact solution after a certain time. Realize also that larger orders of $p$ are needed to get accurate results of the standard deviation than for the expectation (statistical moments of order 2 are harder to approximate than moments of order 1). For $p=3$ and $p=4$, the approximate expectations agree up to 
time $t=7$, whereas the standard deviations up to $t=4.5$. For $p=2$ and $p=3$, similar means are obtained until $t=6$, and similar standard deviations up to $t=4$. Notice that the convergence deteriorates for $p=1$ : the results for $p=1$ and $p=2$ agree until $t=4$ for the expectation, but up to instant $t=1.5$ for the standard deviation. As $p$ grows, the approximation of the statistics will improve for larger $t$.

Table 1: Approximation of $\mathbb{E}[X(t)]$. Example 4.1, assuming independent random data.

\begin{tabular}{|c|c|c|c|c|c|c|c|}
\hline \hline$t$ & $\mathrm{gPC} p=3$ & $\mathrm{gPC} p=4$ & Fröb. $N=3$ & Fröb. $N=5$ & dishonest & MC 50, 000 & MC 100,000 \\
\hline 0.00 & 1 & & 1 & 1 & 1 & 0.99701 & 1.00138 \\
\hline 0.25 & 1.49870 & 1.49870 & 1.49870 & 1.49870 & 1.49870 & 1.49519 & 1.49976 \\
\hline 0.50 & 1.98752 & 1.98752 & 1.98752 & 1.98752 & 1.98752 & 1.98353 & 1.98829 \\
\hline 0.75 & 2.45108 & 2.45108 & 2.45108 & 2.45108 & 2.45102 & 2.44667 & 2.45160 \\
\hline 1.00 & 2.86856 & 2.86856 & 2.86856 & 2.86856 & 2.86818 & 2.86383 & 2.86893 \\
\hline 1.25 & 3.21494 & 3.21494 & 3.21494 & 3.21494 & 3.21339 & 3.21008 & 3.21534 \\
\hline 1.50 & 3.46310 & 3.46310 & 3.46310 & 3.46310 & 3.45812 & 3.45831 & 3.46376 \\
\hline 1.75 & 3.58660 & 3.58660 & 3.58660 & 3.58660 & 3.57340 & 3.58215 & 3.58784 \\
\hline 2.00 & 3.56335 & 3.56335 & 3.56336 & 3.56335 & 3.53286 & 3.55948 & 3.56552 \\
\hline \hline
\end{tabular}

Table 2: Approximation of $\mathbb{V}[X(t)]$. Example 4.1, assuming independent random data.

\begin{tabular}{|c|c|c|c|c|c|c|}
\hline \hline$t$ & gPC $p=3$ & gPC $p=4$ & Fröb. $N=3$ & Fröb. $N=5$ & MC 50, 000 & MC 100, 000 \\
\hline 0.00 & 1 & 1 & 1 & 1 & 0.99610 & 0.99530 \\
\hline 0.25 & 1.06035 & 1.06035 & 1.06035 & 1.06035 & 1.05902 & 1.05642 \\
\hline 0.50 & 1.23142 & 1.23142 & 1.23142 & 1.23142 & 1.23408 & 1.22793 \\
\hline 0.75 & 1.49261 & 1.49261 & 1.49261 & 1.49261 & 1.50041 & 1.48944 \\
\hline 1.00 & 1.81392 & 1.81392 & 1.81392 & 1.81392 & 1.82744 & 1.81127 \\
\hline 1.25 & 2.15870 & 2.15870 & 2.15870 & 2.15870 & 2.17768 & 2.15721 \\
\hline 1.50 & 2.49379 & 2.49379 & 2.49379 & 2.49379 & 2.51690 & 2.49462 \\
\hline 1.75 & 2.80560 & 2.80560 & 2.80560 & 2.80560 & 2.83029 & 2.81030 \\
\hline 2.00 & 3.11530 & 3.11530 & 3.11530 & 3.11530 & 3.13783 & 3.12559 \\
\hline \hline
\end{tabular}

Table 3: Approximation of $\operatorname{Cov}[X(t), X(s)]$ via adapted gPC with $p=3$ and $p=4$. Example 4.1, assuming independent random data.

\begin{tabular}{|c|c|c|c|c|c|c|c|c|c|}
\hline \hline$t-s$ & 0 & 0.25 & 0.5 & 0.75 & 1 & 1.25 & 1.5 & 1.75 & 2 \\
\hline 0 & 1. & 0.998959 & 0.991684 & 0.972072 & 0.934436 & 0.873965 & 0.787323 & 0.673299 & 0.533429 \\
\hline 0.25 & 0.998959 & 1.06035 & 1.11507 & 1.15586 & 1.17516 & 1.16565 & 1.12103 & 1.03694 & 0.911972 \\
\hline 0.5 & 0.991684 & 1.11507 & 1.23142 & 1.33242 & 1.40874 & 1.45067 & 1.44906 & 1.39647 & 1.28856 \\
\hline 0.75 & 0.972072 & 1.15586 & 1.33242 & 1.49261 & 1.62561 & 1.7196 & 1.76286 & 1.74509 & 1.65909 \\
\hline 1 & 0.934436 & 1.17516 & 1.40874 & 1.62561 & 1.81392 & 1.96032 & 2.05099 & 2.07321 & 2.01713 \\
\hline 1.25 & 0.873965 & 1.16565 & 1.45067 & 1.7196 & 1.96032 & 2.1587 & 2.2997 & 2.3688 & 2.35387 \\
\hline 1.5 & 0.787323 & 1.12103 & 1.44906 & 1.76286 & 2.05099 & 2.2997 & 2.49379 & 2.61793 & 2.65822 \\
\hline 1.75 & 0.673299 & 1.03694 & 1.39647 & 1.74509 & 2.07321 & 2.3688 & 2.61793 & 2.8056 & 2.91699 \\
\hline 2 & 0.533429 & 0.911972 & 1.28856 & 1.65909 & 2.01713 & 2.35387 & 2.65822 & 2.91699 & 3.1153 \\
\hline \hline
\end{tabular}

By using the random Fröbenius method from [16], an example of Airy's differential equation with dependent random inputs is performed. It is set $\left(A, Y_{0}, Y_{1}\right)$ to have a multivariate Gaussian distribution, with 
Figure 1: Expectation and confidence interval for the solution stochastic process, for orders of basis $p=1,2,3$, 4. Example 4.1, assuming independent random data.

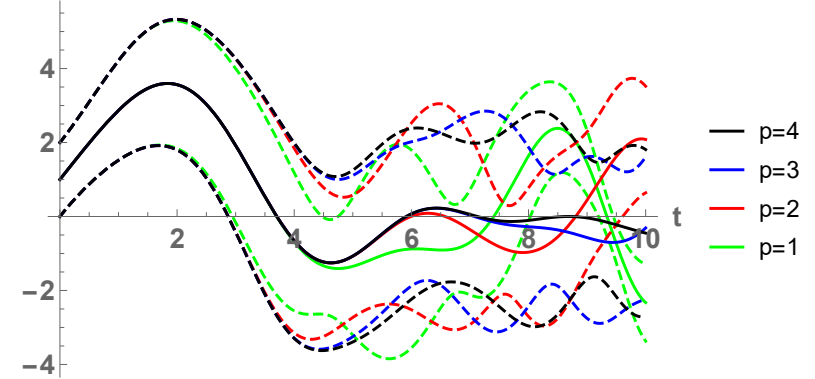

mean vector and covariance matrix given by

$$
\mu=\left(\begin{array}{c}
0.4 \\
1 \\
2
\end{array}\right), \quad \Sigma=\left(\begin{array}{ccc}
0.04 & 0.0001 & -0.05 \\
0.0001 & 1 & 0.5 \\
-0.005 & 0.5 & 1
\end{array}\right),
$$

respectively. In Table 4, Table 5 and Table 6, the results obtained via adaptive gPC with $p=3, p=4$ (dependent case) and [16] are shown. Adaptive gPC converges for small order of basis $p$.

In Figure 2, we analyze the convergence of gPC expansions by depicting the expectation (solid line) and confidence interval (dashed lines) for $X(t)$, where the confidence interval is constructed as mean \pm deviation. Analogous comments to those from Figure 1 apply in this case again. For orders $p=3$ and $p=4$, the expectations agree up to time $t=6$, while the standard deviations coincide until $t=4.6$. For $p=2$ and $p=3$, the means are similar until $t=6$, whereas the dispersion estimates start separating from $t=3.8$. Finally, for $p=1$ and $p=2$, the approximations for the average statistic coincide till $t=4.5$, and for the deviation statistic until $t=2.5$.

Table 4: Approximation of $\mathbb{E}[X(t)]$. Example 4.1, assuming dependent random data.

\begin{tabular}{|c|c|c|c|c|c|c|c|}
\hline \hline$t$ & $\mathrm{gPC} p=3$ & $\mathrm{gPC} p=4$ & Fröb. $N=4$ & Fröb. $N=5$ & dishonest & MC 50, 000 & MC 100,000 \\
\hline 0.00 & 1 & 1 & 1 & 1 & 1 & 1.00188 & 1.00597 \\
\hline 0.25 & 1.49870 & 1.49870 & 1.49870 & 1.49870 & 1.49870 & 1.50166 & 1.50581 \\
\hline 0.50 & 1.98755 & 1.98755 & 1.98755 & 1.98755 & 1.98752 & 1.99156 & 1.99575 \\
\hline 0.75 & 2.45121 & 2.45121 & 2.45121 & 2.45121 & 2.45102 & 2.45622 & 2.46041 \\
\hline 1.00 & 2.86895 & 2.86895 & 2.86895 & 2.86895 & 2.86818 & 2.87485 & 2.87900 \\
\hline 1.25 & 3.21589 & 3.21589 & 3.21589 & 3.21589 & 3.21339 & 3.22247 & 3.22656 \\
\hline 1.50 & 3.46503 & 3.46503 & 3.46503 & 3.46503 & 3.45812 & 3.47198 & 3.47601 \\
\hline 1.75 & 3.59010 & 3.59010 & 3.59010 & 3.59010 & 3.57340 & 3.59700 & 3.60101 \\
\hline 2.00 & 3.56914 & 3.56914 & 3.56915 & 3.56914 & 3.53286 & 3.57546 & 3.57949 \\
\hline \hline
\end{tabular}

Example 4.2. Consider the random differential equation

$$
\left\{\begin{array}{l}
\ddot{X}(t)+\left(\gamma_{1}+\gamma_{2} t\right) \dot{X}(t)+\left(\eta_{1}+t\right) X(t)=\xi_{1} \cos (t)+g(t), t \in \mathbb{R}, \\
X(0)=Y_{0}, \\
\dot{X}(0)=Y_{1},
\end{array}\right.
$$

where $\gamma_{1} \sim \operatorname{Poisson}(3), \gamma_{2} \sim \operatorname{Uniform}(0,1), \eta_{1} \sim \operatorname{Gamma}(2,2), Y_{0}=-1, Y_{1} \sim \operatorname{Exponential}(4), \xi_{1} \sim$ Uniform $(-8,2)$ and $g(t)=\mathrm{e}^{-1 / t} 1_{(0, \infty)}(t)$. 
Table 5: Approximation of $\mathbb{V}[X(t)]$. Example 4.1, assuming dependent random data.

\begin{tabular}{|c|c|c|c|c|c|c|}
\hline \hline$t$ & $\mathrm{gPC} p=3$ & $\mathrm{gPC} p=4$ & Fröb. $N=3$ & Fröb. $N=4$ & MC 50,000 & MC 100, 000 \\
\hline 0.00 & 1 & 1 & 1 & 1 & 0.999223 & 0.99992 \\
\hline 0.25 & 1.30997 & 1.30997 & 1.30997 & 1.30997 & 1.30713 & 1.30991 \\
\hline 0.50 & 1.72535 & 1.72535 & 1.72535 & 1.72535 & 1.71989 & 1.72525 \\
\hline 0.75 & 2.21241 & 2.21241 & 2.21241 & 2.21241 & 2.20395 & 2.21230 \\
\hline 1.00 & 2.72122 & 2.72122 & 2.72122 & 2.72122 & 2.70957 & 2.72125 \\
\hline 1.25 & 3.19236 & 3.19236 & 3.19236 & 3.19236 & 3.17745 & 3.19283 \\
\hline 1.50 & 3.57361 & 3.57361 & 3.57361 & 3.57361 & 3.55537 & 3.57484 \\
\hline 1.75 & 3.84459 & 3.84459 & 3.84454 & 3.84458 & 3.82262 & 3.84669 \\
\hline 2.00 & 4.04087 & 4.04087 & 4.04090 & 4.04086 & 4.01420 & 4.04342 \\
\hline \hline
\end{tabular}

Table 6: Approximation of $\operatorname{Cov}[X(t), X(s)]$ via adapted gPC with $p=3$ and $p=4$. Example 4.1, assuming dependent random data.

\begin{tabular}{|c|c|c|c|c|c|c|c|c|c|}
\hline \hline$t-s$ & 0 & 0.25 & 0.5 & 0.75 & 1 & 1.25 & 1.5 & 1.75 & 2 \\
\hline 0 & 1. & 1.12389 & 1.24064 & 1.34181 & 1.41793 & 1.45914 & 1.45614 & 1.40144 & 1.29068 \\
\hline 0.25 & 1.12389 & 1.30997 & 1.48771 & 1.64683 & 1.77533 & 1.86031 & 1.88919 & 1.85125 & 1.7394 \\
\hline 0.5 & 1.24064 & 1.48771 & 1.72535 & 1.94152 & 2.12187 & 2.25061 & 2.31202 & 2.29226 & 2.18163 \\
\hline 0.75 & 1.34181 & 1.64683 & 1.94152 & 2.21241 & 2.4431 & 2.61534 & 2.71062 & 2.71235 & 2.60832 \\
\hline 1 & 1.41793 & 1.77533 & 2.12187 & 2.4431 & 2.72122 & 2.93619 & 3.06742 & 3.09609 & 3.00785 \\
\hline 1.25 & 1.45914 & 1.86031 & 2.25061 & 2.61534 & 2.93619 & 3.19236 & 3.36219 & 3.42552 & 3.36639 \\
\hline 1.5 & 1.45614 & 1.88919 & 2.31202 & 2.71062 & 3.06742 & 3.36219 & 3.57361 & 3.68135 & 3.66862 \\
\hline 1.75 & 1.40144 & 1.85125 & 2.29226 & 2.71235 & 3.09609 & 3.42552 & 3.68135 & 3.84459 & 3.89856 \\
\hline 2 & 1.29068 & 1.7394 & 2.18163 & 2.60832 & 3.00785 & 3.36639 & 3.66862 & 3.89856 & 4.04087 \\
\hline \hline
\end{tabular}

Figure 2: Expectation and confidence interval for the solution stochastic process, for orders of basis $p=1,2,3,4$. Example 4.1, assuming dependent random data.

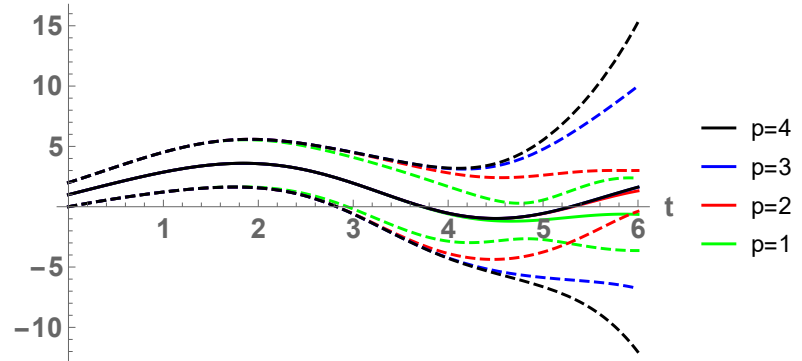

Proposition 2.1 ensures the existence and uniqueness of a sample path solution. To apply Proposition 2.2, one would need to truncate the supports of $\gamma_{1}$ and $\eta_{1}$. These truncations can be constructed on intervals as large as desired, in order to maintain the results.

The input random variables $\zeta_{1}=\gamma_{1}, \zeta_{2}=\gamma_{2}, \zeta_{3}=\eta_{1}, \zeta_{4}=\zeta_{1}$ and $\zeta_{5}=Y_{1}$ are assumed to be independent. The involved functions are $a_{1}(t)=1, a_{2}(t)=t, b_{1}(t)=1, b_{2}(t)=t, c_{0}(t)=g(t)$ and $c_{1}(t)=\cos (t)$. Notice that $C(t)$ is not an analytic stochastic process, because $g(t)$ is not a real analytic function. The random Fröbenius method is not applicable for the random IVP (15). However, we are going to see that adaptive gPC (independent case) with $p=6$ and $p=7$ provides reliable approximations of the expectation and covariance of $X(t)$. We will compare the results with Monte Carlo simulation. In Table 7, Table 8 and Table 9 we show the estimates obtained. 
In Figure 3, we focus on the convergence of gPC expansions. We depict the estimates of the expectations (solid line) and confidence intervals (dashed lines), with the rule mean \pm deviation, for orders $p=4,5,6,7$. Note that convergence is achieved for $t \in[0,10]$.

Table 7: Approximation of $\mathbb{E}[X(t)]$. Example 4.2, assuming independent random data.

\begin{tabular}{|c|c|c|c|c|c|}
\hline \hline$t$ & gPC $p=6$ & gPC $p=7$ & dishonest & MC 50, 000 & MC 100, 000 \\
\hline 0.00 & -1 & -1 & -1 & -1 & -1 \\
\hline 0.25 & -0.930972 & -0.930972 & -0.931372 & -0.931035 & -0.930364 \\
\hline 0.50 & -0.855779 & -0.855779 & -0.852372 & -0.855937 & -0.854386 \\
\hline 0.75 & -0.780021 & -0.780021 & -0.759103 & -0.780573 & -0.778022 \\
\hline 1.00 & -0.700758 & -0.700758 & -0.647653 & -0.702042 & -0.698437 \\
\hline 1.25 & -0.609156 & -0.609156 & -0.518169 & -0.611266 & -0.606832 \\
\hline 1.50 & -0.496445 & -0.496446 & -0.374486 & -0.499156 & -0.494407 \\
\hline 1.75 & -0.359632 & -0.359635 & -0.222874 & -0.362532 & -0.358036 \\
\hline 2.00 & -0.203726 & -0.203737 & -0.070806 & -0.206408 & -0.202560 \\
\hline \hline
\end{tabular}

Table 8: Approximation of $\mathbb{V}[X(t)]$. Example 4.2, assuming independent random data.

\begin{tabular}{|c|c|c|c|c|}
\hline \hline$t$ & gPC $p=6$ & gPC $p=7$ & MC 50, 000 & MC 100, 000 \\
\hline 0.00 & 0 & 0 & 0 & 0 \\
\hline 0.25 & 0.0114271 & 0.0114271 & 0.0115378 & 0.0114953 \\
\hline 0.50 & 0.0897916 & 0.0897916 & 0.0904703 & 0.090160 \\
\hline 0.75 & 0.236135 & 0.236136 & 0.237288 & 0.237066 \\
\hline 1.00 & 0.371625 & 0.371639 & 0.372899 & 0.373058 \\
\hline 1.25 & 0.426921 & 0.427021 & 0.428690 & 0.428342 \\
\hline 1.50 & 0.388485 & 0.388899 & 0.391305 & 0.38978 \\
\hline 1.75 & 0.289622 & 0.290720 & 0.293631 & 0.291429 \\
\hline 2.00 & 0.182954 & 0.184922 & 0.187906 & 0.185917 \\
\hline \hline
\end{tabular}

Table 9: Approximation of $\operatorname{Cov}[X(t), X(s)]$ via adapted $\mathrm{gPC}$ with $p=7$. Example 4.2, assuming independent random data.

\begin{tabular}{|c|c|c|c|c|c|c|c|c|c|}
\hline \hline$t-s$ & 0 & 0.25 & 0.5 & 0.75 & 1 & 1.25 & 1.5 & 1.75 & 2 \\
\hline 0 & 0 & 0 & 0 & 0 & 0 & 0 & 0 & 0 & 0 \\
\hline 0.25 & 0 & 0.0114271 & 0.0310997 & 0.0486065 & 0.0585142 & 0.0597207 & 0.0537473 & 0.042977 & 0.0295893 \\
\hline 0.5 & 0 & 0.0310997 & 0.0897916 & 0.14431 & 0.176903 & 0.182770 & 0.165621 & 0.132554 & 0.0905903 \\
\hline 0.75 & 0 & 0.0486065 & 0.14431 & 0.236136 & 0.293929 & 0.307634 & 0.281495 & 0.226547 & 0.154877 \\
\hline 1 & 0 & 0.0585142 & 0.176903 & 0.293929 & 0.371639 & 0.394991 & 0.366485 & 0.29839 & 0.206021 \\
\hline 1.25 & 0 & 0.0597207 & 0.182770 & 0.307634 & 0.394991 & 0.427021 & 0.403260 & 0.334311 & 0.235733 \\
\hline 1.5 & 0 & 0.0537473 & 0.165621 & 0.281495 & 0.366485 & 0.403260 & 0.388899 & 0.330575 & 0.241178 \\
\hline 1.75 & 0 & 0.042977 & 0.132554 & 0.226547 & 0.29839 & 0.334311 & 0.330575 & 0.29072 & 0.223109 \\
\hline 2 & 0 & 0.0295893 & 0.0905903 & 0.154877 & 0.206021 & 0.235733 & 0.241178 & 0.223109 & 0.184922 \\
\hline \hline
\end{tabular}


Figure 3: Expectation and confidence interval for the solution stochastic process, for orders of basis $p=4,5,6,7$. Example 4.2, assuming independent random data.

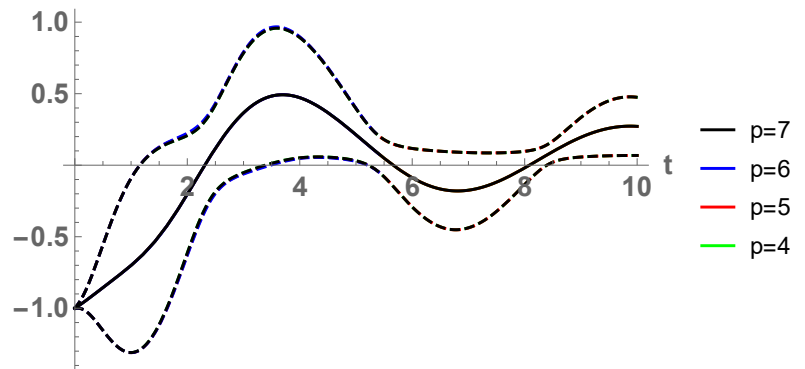

Example 4.3. Consider the random differential equation

$$
\left\{\begin{array}{l}
\ddot{X}(t)+B(t) X(t)=C, t \in[0,1], \\
X(0)=Y_{0}, \\
\dot{X}(0)=Y_{1},
\end{array}\right.
$$

where $B(t)$ is a standard Brownian motion on [0,1], $C \sim$ Poisson(2), and the initial conditions are distributed as $Y_{0} \sim \operatorname{Beta}(1 / 2,1 / 2)$ and $Y_{1}=0$. These random inputs are assumed to be independent.

This stochastic system has a unique solution in the sample path sense, by Proposition 2.1. In principle, one cannot ensure the existence of a mean square solution, since the sample paths of Brownian motion are not bounded.

Consider the Karhunen-Loève expansion of Brownian motion [25, p. 216]:

$$
B(t)=\sum_{j=1}^{\infty} \frac{\sqrt{2}}{\left(j-\frac{1}{2}\right) \pi} \sin \left(\left(j-\frac{1}{2}\right) \pi t\right) \xi_{j},
$$

where $\xi_{1}, \xi_{2}, \ldots$ are independent and $\operatorname{Normal}(0,1)$ random variables. The series is understood in $\mathrm{L}^{2}([0,1] \times$ $\Omega$ ). We truncate the Karhunen-Loève expansion so that $B(t)$ will have the form in (2). If we take $d_{B}=7$, we are capturing more than $97 \%$ of the total variance of $X$. Thus, we take

$$
B(t)=\sum_{j=1}^{7} \frac{\sqrt{2}}{\left(j-\frac{1}{2}\right) \pi} \sin \left(\left(j-\frac{1}{2}\right) \pi t\right) \xi_{j} .
$$

The random inputs become $\zeta_{1}=\xi_{1}, \ldots, \zeta_{7}=\xi_{7}, \zeta_{8}=C$ and $\zeta_{9}=Y_{0}$, with functions $b_{j}(t)=\frac{\sqrt{2}}{(j-1 / 2) \pi} \sin ((j-$ $1 / 2) \pi t), 1 \leq j \leq 7$, and $c_{1}(t)=1$.

Notice that, if one truncates $\xi_{1}, \ldots, \xi_{7}$ to a large but bounded support, Proposition 2.2 entails that there exists a solution stochastic process in the mean square sense.

In Table 10, Table 11 and Table 12, we show the results obtained by adaptive gPC with $p=2, p=3$ and Monte Carlo simulation. Similar estimates are obtained for $p=2$ and $p=3$, which agrees with the convergence of gPC-based representations.

In Figure 4, we show graphically the convergence of gPC expansions on $[0,1]$ : we plot the approximate expectations (solid line) and confidence intervals (dashed lines) for $X(t)$, where the confidence interval is constructed as mean \pm deviation. For $p=1,2,3$, no differences in the estimates are observed.

\section{Conclusions}

In this paper, we have quantified computationally the uncertainty of random non-autonomous second-order linear differential equations via adaptive $\mathrm{gPC}$. After reviewing adaptive $\mathrm{gPC}$ from the extant literature, we have 
Table 10: Approximation of $\mathbb{E}[X(t)]$. Example 4.3, assuming independent random data.

\begin{tabular}{|c|c|c|c|c|c|}
\hline \hline$t$ & gPC $p=2$ & gPC $p=3$ & dishonest & MC 50,000 & MC 100, 000 \\
\hline 0.00 & 0.5 & 0.5 & 0.5 & 0.499302 & 0.499056 \\
\hline 0.25 & 0.562504 & 0.562504 & 0.5625 & 0.561732 & 0.561438 \\
\hline 0.50 & 0.75014 & 0.75014 & 0.75 & 0.749196 & 0.748740 \\
\hline 0.75 & 1.06365 & 1.06365 & 1.0625 & 1.06245 & 1.06179 \\
\hline 1.00 & 1.50536 & 1.50536 & 1.5 & 1.50396 & 1.50311 \\
\hline \hline
\end{tabular}

Table 11: Approximation of $\mathbb{V}[X(t)]$. Example 4.3, assuming independent random data.

\begin{tabular}{|c|c|c|c|c|}
\hline \hline$t$ & $\mathrm{gPC} p=2$ & $\mathrm{gPC} p=3$ & $\mathrm{MC} 50,000$ & $\mathrm{MC} 100,000$ \\
\hline 0.00 & 0.125 & 0.125 & 0.124849 & 0.124826 \\
\hline 0.25 & 0.126974 & 0.126974 & 0.126883 & 0.126841 \\
\hline 0.50 & 0.157008 & 0.157008 & 0.157267 & 0.157033 \\
\hline 0.75 & 0.290263 & 0.290265 & 0.291811 & 0.290766 \\
\hline 1.00 & 0.664551 & 0.664592 & 0.670067 & 0.666466 \\
\hline \hline
\end{tabular}

Table 12: Approximation of $\operatorname{Cov}[X(t), X(s)]$ via adapted $\mathrm{gPC}$ with $p=3$. Example 4.3, assuming independent random data.

\begin{tabular}{|c|c|c|c|c|c|}
\hline \hline$t-s$ & 0 & 0.25 & 0.5 & 0.75 & 1 \\
\hline 0 & 0.125 & 0.125001 & 0.125033 & 0.125248 & 0.126043 \\
\hline 0.25 & 0.125001 & 0.126974 & 0.132949 & 0.143104 & 0.157885 \\
\hline 0.5 & 0.125033 & 0.132949 & 0.157008 & 0.197634 & 0.255578 \\
\hline 0.75 & 0.125248 & 0.143104 & 0.197634 & 0.290265 & 0.422829 \\
\hline 1 & 0.126043 & 0.157885 & 0.255578 & 0.422829 & 0.664592 \\
\hline \hline
\end{tabular}

Figure 4: Expectation and confidence interval for the solution stochastic process, for orders of basis $p=1,2,3$. Example 4.3, assuming independent random data.

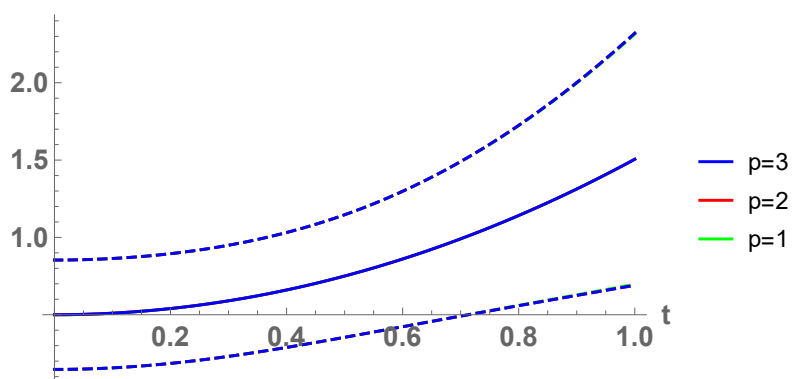


provided a methodology and an algorithm to approximate computationally the expectation and covariance of the solution stochastic process. The hypotheses from our algorithm allow both independent and dependent random parameter inputs, being both absolutely continuous or discrete with infinitely many point masses. The generality of our computational results allows the random input coefficients to be truncated random power series or truncated Karhunen-Loève expansions. The former case permits comparing our methodology with the random Fröbenius method, an approach already used in the literature with particular random second-order linear differential equations, and Monte Carlo simulation. A wide variety of examples show that adaptive gPC becomes successful quantifying the uncertainty of random non-autonomous second-order linear differential equations, even when the random Fröbenius method is not applicable.

Acknowledgement: This work has been supported by the Spanish Ministerio de Economía y Competitividad grant MTM2017-89664-P. Marc Jornet acknowledges the doctorate scholarship granted by Programa de Ayudas de Investigación y Desarrollo (PAID), Universitat Politècnica de València. The authors are grateful for the valuable comments raised by the reviewer, which have improved the final version of the paper.

Conflict of Interest Statement: The authors declare that there is no conflict of interests regarding the publication of this article.

\section{References}

[1] Strand J.L., Random Ordinary Differential Equations, J. Differ. Equ., 1970, 7, 538-553.

[2] Soong T.T., Random Differential Equations in Science and Engineering, 1973, New York: Academic Press.

[3] Xiu D., Numerical Methods for Stochastic Computations. A Spectral Method Approach, 2010, Princeton University Press.

[4] Xiu D., Karniadakis G.E, The Wiener-Askey polynomial chaos for stochastic differential equations, SIAM J. Sci. Comput., 2002, 24 (2), 619-644.

[5] Williams M.M.R., Polynomial chaos functions and stochastic differential equations, Ann. Nucl. Energy, 2006, 33 (9), 774-785.

[6] Chen-Charpentier B.M., Stanescu D., Epidemic models with random coefficients, Math. Comput. Model., 2010, 52 (7-8), 1004-1010.

[7] Chen-Charpentier B.M., Cortés J.C., Licea J.A., Romero J.V., Roselló M.D., Santonja F.J., Villanueva R.J., Constructing adaptive generalized polynomial chaos method to measure the uncertainty in continuous models: A computational approach, Math. Comput. Simulat., 2015, 109, 113-129.

[8] Cortés J.C., Romero J.V., Roselló M.D., Villanueva R.J., Improving adaptive generalized polynomial chaos method to solve nonlinear random differential equations by the random variable transformation technique, Commun. Nonlinear Sci. Numer. Simulat., 2017, 50, 1-15.

[9] Cortés J.C., Romero J.V., Roselló M.D., Santonja F.J., Villanueva R.J., Solving Continuous Models with Dependent Uncertainty: A Computational Approach, Abstr. Appl. Anal., 2013.

[10] Cortés J.C., Navarro-Quiles A., Romero J.V., Roselló M.D., Probabilistic solution of random autonomous first-order linear systems of ordinary differential equations, Rom. Rep. Phys., 2016, 68 (4), 1397-1406.

[11] Gottlieb D., Xiu D., Galerkin method for wave equations with uncertain coefficients, Commun. Comput. Phys., 2008, 3 (2), 505-518.

[12] Ernst O.G., Mugler A., Starkloff H.J., Ullmann E., On the convergence of generalized polynomial chaos expansions, ESAIM-Math. Model. Num., 2012, 46 (2), 317-339.

[13] Shi W., Zhang C., Error analysis of generalized polynomial chaos for nonlinear random ordinary differential equations, Appl. Numer. Math., 2012, 62 (12), 1954-1964.

[14] Shi W., Zhang C., Generalized polynomial chaos for nonlinear random delay differential equations, Appl. Numer. Math., 2017, 115, 16-31.

[15] Calatayud J., Cortés J.C., Jornet M., On the convergence of adaptive gPC for non-linear random difference equations: Theoretical analysis and some practical recommendations, J. Nonlinear Sci. App., 2018, 11 (9), 1077-1084.

[16] Cortés J.C., Jódar L., Camacho F., Villafuerte L., Random Airy type differential equations: Mean square exact and numerical solutions, Comput. Math. Appl., 2010, 60, 1237-1244.

[17] Calbo G., Cortés J.C., Jódar L., Random Hermite differential equations: Mean square power series solutions and statistical properties, Appl. Math. Comput., 2011, 218 (7), 3654-3666.

[18] Calbo G., Cortés J.C., Jódar L., Villafuerte L., Solving the random Legendre differential equation: Mean square power series solution and its statistical functions, Comput. Math. Appl., 2011, 61 (9), 2782-2792. 
[19] Calatayud J., Cortés J.C., Jornet M., Improving the approximation of the first and second order statistics of the response process to the random Legendre differential equation, 2018, arXiv preprint, arXiv:1807.03141.

[20] Cortés J.C., Jódar L., Company R., Villafuerte L., Laguerre random polynomials: definition, differential and statistical properties, Utilitas Mathematica, 2015, 98, 283-295.

[21] Cortés J.C., Jódar L., Villafuerte L., Mean square solution of Bessel differential equation with uncertainties, J. Comput. Appl. Math., 2017, 309 (1), 383-395.

[22] Calatayud J., Cortés J.C., Jornet M., Villafuerte L., Random non-autonomous second order linear differential equations: mean square analytic solutions and their statistical properties, Adv. Differ. Equ., 2018, 392, 1-29.

[23] Calatayud J., Cortés J.C., Jornet M., Some notes to extend the study on random non-autonomous second order linear differential equations appearing in Mathematical Modeling, Math. Comput. Appl., 2018, 23 (4), 76-89.

[24] Golmankhaneh A.K., Porghoveh N.A., Baleanu D., Mean square solutions of second-order random differential equations by using homotopy analysis method, Rom. Rep. Phys., 2013, 65 (2).

[25] Lord G.J., Powell C.E., Shardlow T., An Introduction to Computational Stochastic PDEs, 2014, New York: Cambridge Texts in Applied Mathematics, Cambridge University Press.

[26] Hale J.K., Ordinary Differential Equations (2nd ed.), 1980, Malabar: Robert E. Krieger Publishing Company.

[27] Henderson D., Plaschko P., Stochastic Differential Equations in Science and Engineering, 2006, Singapore: World Scientific.

[28] Vallée O., Soares M., Airy Functions and Applications to Physics, 2004, London: Imperial College Press. 